\title{
ENGEVISTA
}

Página da revista: http://www.uff.br/engevista/seer/

\section{Avaliação das cheias ordinárias no Rio Paraíba do Sul através das vazões mínimas e médias de longo termo}

\author{
Raquel Veloso Ferreira ${ }^{1}$ \\ Mônica de Aquino G. Massera da Hora $^{2}$
}

Resumo: O presente estudo buscou avaliar as cheias ordinárias no estirão do rio Paraíba do Sul através da sua correlação com as vazões Q95\% e MLT. A relevância do estudo deve-se ao fato de que a região é o elo entre as maiores regiões metropolitanas do país e suas águas são um recurso fundamental para diferentes usuários. Além disso, o estudo das cheias ordinárias é importante para auxiliar a demarcação de terrenos marginais por meio da determinação da posição da Linha Média das Enchentes Ordinárias. Foram realizadas duas análises, a primeira utilizou o resultado do conjunto de postos fluviométricos da bacia e a segunda adotou como critério a divisão dos postos em três grupos representativos dos trechos superior, médio e inferior do rio Paraíba do Sul. Os resultados alcançados permitiram inferir que a vazão Q95\% se relaciona muito bem com a MLT e que a vazão QTR=3 anos, representativa das cheias ordinárias, se relaciona melhor com a MLT do que com a Q95\%. Por fim, a divisão do rio Paraíba do Sul em três trechos resulta em melhores ajustes de correlação entre as vazões para o trecho inferior. Mas o melhor resultado global foi aquele que leva em consideração todos os postos fluviométricos, sem a divisão por trechos.

Palavras-chave: Cheias ordinárias, rio Paraíba do Sul, Q95\%, MLT.

${ }^{1}$ UFF - Universidade Federal Fluminense

${ }^{2}$ UFF - Universidade Federal Fluminense 
ISSN: $1415-7314$

ISSN online: $2317-6717$

\begin{abstract}
This study aimed to evaluate the ordinary floods in the Paraíba do Sul river through its correlation with Q95\% and MLT discharges. The relevance of the study is due to the fact that the region is the link between the major metropolitan areas and its waters are a relevant resource for different users. In addition, the study of ordinary floods is important to assist the demarcation of marginal lands by determining the average flood line limit position. Two analyzes were conducted, the first used the results of all of the basin gauge stations and the second adopted the division of the Paraiba do Sul river in three representative groups: upper, middle and bottom stretches. The results allowed to infer that the Q95\% relates very well with the MLT and the QTR $=3$ years, representative of the ordinary floods, relates better with MLT than with Q95\%. Finally, the division of the Paraiba do Sul River in three sections presents better correlation between the discharge adjustments to the lower stretch. But the best overall result was the one that took into account all gauge stations without the division by stretches.
\end{abstract}

Keywords:. Ordinary floods, Paraíba do Sul river, Q95\%, MLT. 


\section{Introdução}

A bacia hidrográfica do rio Paraíba do Sul se estende por territórios pertencentes a três Estados da Região Sudeste, São Paulo, Rio de Janeiro e Minas Gerais. Atualmente, a bacia está localizada em uma das regiões mais habitadas e industrializadas do Brasil, e, de acordo com Marengo e Alves (2005), é responsável por cerca de 10\% do PIB nacional. As águas da bacia são utilizadas por diversos setores tais como geração de energia, abastecimento público, indústria e agropecuária.

O rio Paraíba do Sul é o mais importante da região Sudeste, entretanto o fato da região ser altamente desenvolvida contribui para a existência de problemas relativos à qualidade e disponibilidade de água. A concentração da população e de indústrias no entorno da bacia geram pressões sobre os recursos hídricos, e a crescente demanda aumenta os conflitos pelo uso da água na região, a exemplo do atual conflito na operação dos reservatórios Paraibuna, Santa Branca, Jaguari e Funil e do bombeamento em Santa Cecília (ANA, 2015).

Os recursos hídricos são determinantes na ocupação do solo e desenvolvimento das cidades, porém, a sua exploração sem controle e a ocupação de suas faixas marginais causam degradação fluvial e consequentes problemas como erosão, assoreamento, impactos no uso da água e etc. Dada a importância da bacia do Paraíba do Sul, é necessário que sejam tomadas iniciativas para sua preservação, recuperação e adequada gestão dos recursos hídricos para que a segurança hídrica seja assegurada.

No Brasil, há um grande desafio com a aplicação dos limites ambientais contidos nas normas e legislações ambientais no contexto do planejamento territorial e das políticas públicas, (MIRANDA, 2008). Com o intuito de disciplinar e limitar as interferências antrópicas sobre os corpos hídricos, o Código Florestal e outros diplomas legais contemplam as áreas ribeirinhas como de preservação permanente, determinando larguras mínimas para proteção das mesmas, que no âmbito do Estado do Rio de Janeiro são tratadas como Faixas Marginais de Proteção (FMP) (MIRANDA, 2013).

De acordo com SPU (2001), a demarcação dos terrenos marginais de domínio da União deve ser feita por meio da determinação da posição da Linha Média das Enchentes Ordinárias (LMEO). Para efeito de cálculo da média das enchentes ordinárias, devem ser consideradas as cotas máximas anuais referentes às enchentes com período de recorrência igual a 3 anos.

Neste contexto, o estudo das cheias ordinárias é uma ferramenta fundamental para a preservação dos recursos hídricos de domínio federal, auxiliando também nas questões de demarcação e fiscalização dos bens da União.

O presente estudo visa avaliar as cheias ordinárias no estirão do rio Paraíba do Sul através das vazões Q95\% (vazão com permanência de 95\% do tempo) e MLT (vazão média de longo 
termo). Para alcançar os objetivos propostos, foram utilizados como referência os mesmos postos fluviométricos do estudo de regionalização elaborado por CPRM (2013), excluindo-se os que não estavam disponíveis na base de dados do HidroWeb.

Os valores de Q95\% e MLT foram oriundos pelo software Hidro 1.2 da Agência Nacional de Águas (ANA). A vazão máxima para tempo de recorrência (TR) igual a 3 anos, foi estimada com base na metodologia preconizada em Eletrobras (1999). Ao final, os valores das vazões estimadas foram relacionados entre si.

\section{Materiais e métodos}

\section{Estudo de Regionalização desenvolvido pela CPRM}

Os valores de Q95\% apresentados em CPRM (2013) referem-se aos estimados para cada posto fluviométrico a partir da série de vazões médias diárias, bem como àqueles calculados a partir das equações de regionalização definidas para cada região de estudo. A vazão Q95\% foi calculada através de equações de regressão em função das variáveis áreas de drenagem e/ou precipitação média anual. Foram coletados o maior número possível de dados consistidos e préselecionadas as estações fluviométricas com mais de 5 anos de dados de vazão. Calcularam-se ainda indicadores hidrológicos regionais e características estatísticas para análise de sua variabilidade espacial, visando à definição das regiões hidrológica e estatisticamente homogêneas. A partir dos resultados das análises de regressão, foram definidas 20 regiões homogêneas e uma indefinida. Esta última abrange a região da sub-bacia do rio Piraí, que é altamente regulada pela presença de vários reservatórios e estações elevatórias que promovem a transposição de vazões e descaracterizam completamente a bacia (CPRM, 2013).

\section{Estimativa das vazões Q95\% e MLT}

Para o presente estudo, foram consideradas 147 estações fluviométricas adotadas em CPRM (2013), divididas em 21 diferentes regiões homogêneas. Dos 147 postos fluviométricos, apenas 104 estavam disponíveis no banco de dados Hidroweb da Agência Nacional de Águas (ANA). Assim, em virtude da ausência de informações das séries de dados consistidos, $28 \%$ dos postos fluviométricos foram eliminados.

As vazões Q95\% e MLT foram calculadas a partir das séries históricas de vazões diárias das estações fluviométricas extraídas do banco de dados Hidroweb, utilizando o software Hidro 1.2, também disponível na página da ANA.

\section{Cálculo das cheias ordinárias}

Para o cálculo das cheias ordinárias, representativas das vazões máximas com tempo de recorrência (TR) igual a 3 anos, adotou-se a metodologia preconizada em Eletrobras (1999). Para 
a definição das cheias de projeto, foram utilizadas duas distribuições: exponencial de dois parâmetros (estimada pelo método dos momentos), sempre que a assimetria da amostra for superior a 1,5, e Gumbel (extremos do tipo I), para assimetrias amostrais inferiores a 1,5.

O quantil de projeto $\left(\mathrm{x}_{\mathrm{TR}}\right)$ para a distribuição exponencial de dois parâmetros, associado ao tempo de recorrência, é expresso por:

onde:

$$
\begin{gathered}
\mathrm{x}_{\mathrm{TR}}=\mathrm{x}_{\mathrm{O}}-\beta \cdot \ln \cdot\left(\frac{1}{\mathrm{TR}}\right) \\
\mathrm{x}_{\mathrm{O}}=\overline{\mathrm{x}}-\mathrm{s} \\
\beta=\mathrm{s}
\end{gathered}
$$

$\mathrm{x}_{\mathrm{o}}$ e $\beta$ parâmetros da distribuição.

$\mathrm{s}$ desvio padrão da amostra.

$\bar{x}$ média da amostra.

O quantil de projeto ( $\left.\mathrm{x}_{\mathrm{TR}}\right)$ para a distribuição Gumbel, associado ao tempo de recorrência, é expresso por:

$$
\begin{gathered}
\mathrm{x}_{\mathrm{TR}}=\mu-\alpha \cdot\left(\ln \cdot\left(-\ln \cdot\left(1-\frac{1}{\mathrm{TR}}\right)\right)\right) \\
\alpha=0,78 \cdot s \\
\mu=\bar{x}-0,577 \cdot \alpha
\end{gathered}
$$

onde:

$\alpha$ e $\mu$ parâmetros da distribuição.

s desvio padrão da amostra.

$\bar{x}$ média da amostra.

\section{Resultados e discussão}

A Tabela 1, em anexo ao final do artigo, relaciona os resultados alcançados para as vazões Q95\%, MLT e QTR= 3 anos.

Para uma melhor visualização dos resultados obtidos, foram feitos gráficos comparando as variáveis de duas em duas, como pode ser observado nas Figuras de 1 a 3.

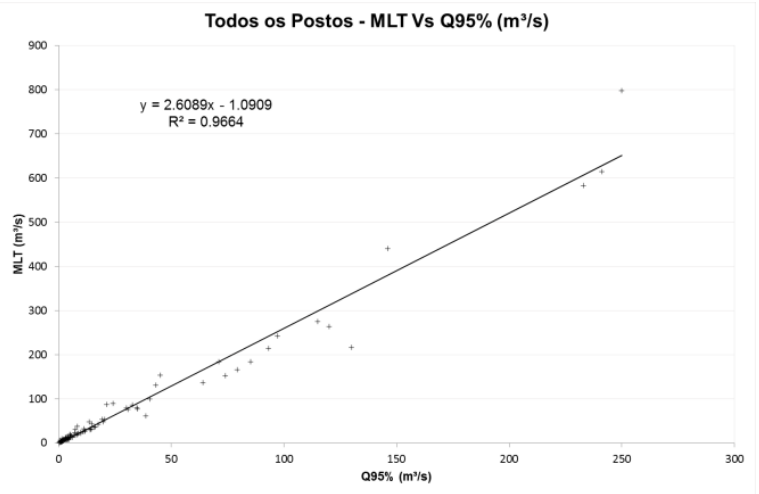

Figura 1. Correlação entre a MLT e a Q95\% para todos os postos 


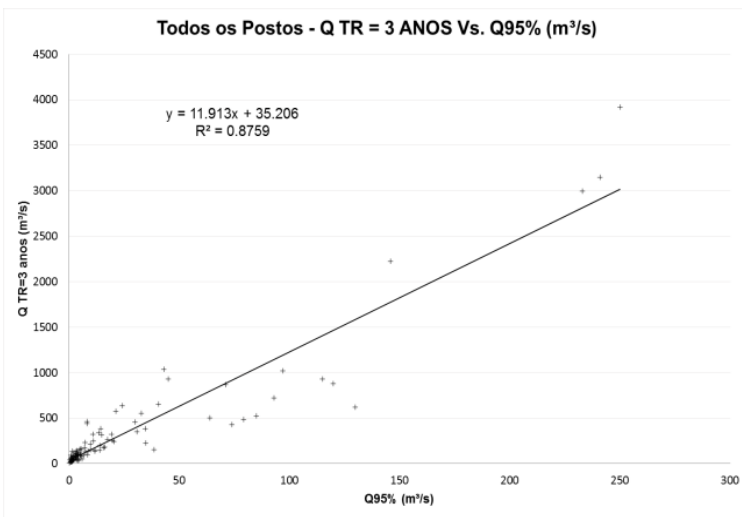

Figura 2. Correlação entre a QTR=3anos e a Q95\% para todos os postos

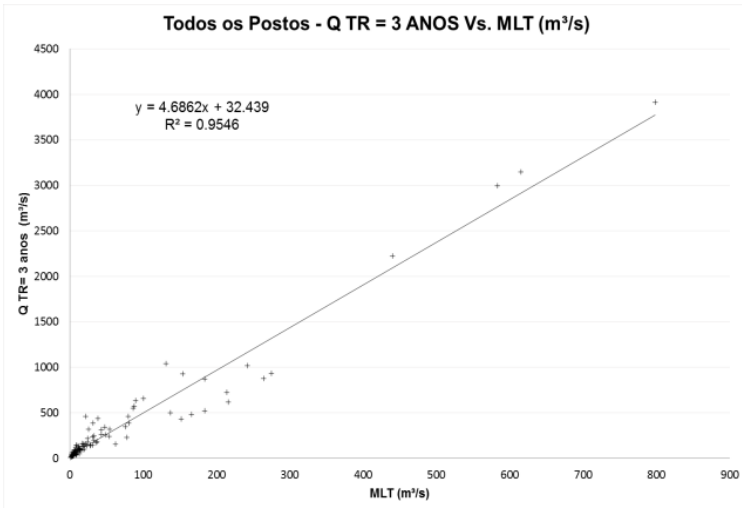

Figura 3. Correlação entre a QTR=3anos e a MLT para todos os postos

Da análise dos gráficos, pode-se observar que os resultados para todos os postos fluviométricos adotados no estudo foram satisfatórios, pois os gráficos de Q95\% versus MLT e MLT versus $Q T R=3$ anos apresentaram valores de coeficiente de determinação $\left(r^{2}\right)$ acima de 0,95 , Já o valor do gráfico $\mathrm{Q} 95 \%$ versus $\mathrm{QTR}=3$ anos apresentou valor de ajuste menor, com $\mathrm{r}^{2}$ igual a 0,8759 .

Foi realizada nova análise adotando-se como critério a divisão dos postos em três grupos: grupo 1, denominado trecho superior que corresponde ao estirão do rio Paraíba do Sul das nascentes até a UHE Funil; grupo 2, denominado de trecho médio, que corresponde ao estirão do rio Paraíba do Sul a jusante da UHE Funil até a confluência dos rios Paraibuna Mineiro e Piabanha; e grupo 3, denominado de trecho inferior, que corresponde ao estirão do rio Paraíba do Sul a jusante da confluência com os rios Paraibuna Mineiro e Piabanha até a foz.

No grupo 1 foram incluídos os postos fluviométricos Estrada do Cunha (58030000) até Queluz (58235100). Os resultados das correlações são apresentados nas Figuras 4 a 6. 


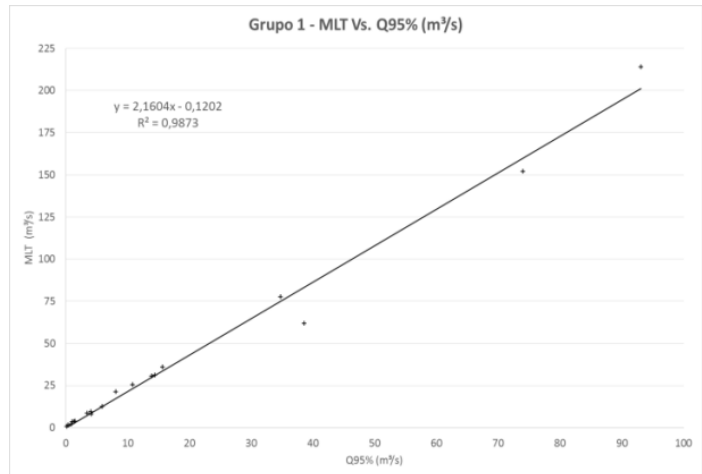

Figura 4. MLT versus Q95\% para o grupo 1

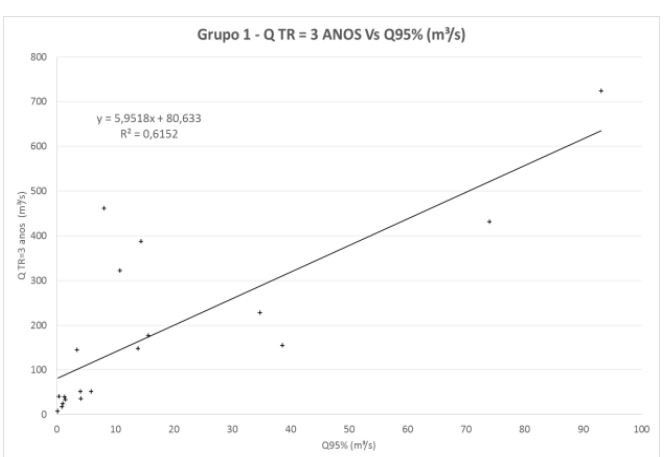

Figura 5. Correlação entre a QTR=3anos e a Q95\% para o grupo 1

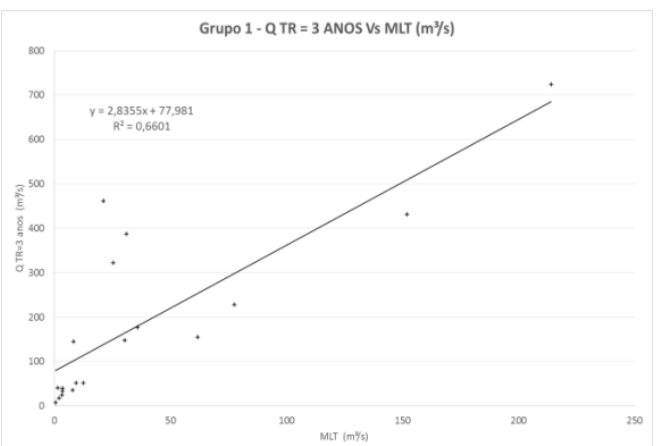

Figura 6. Correlação entre a QTR=3 anos e a MLT para o grupo 1.

No grupo 2 foram incluídos os postos fluviométricos Itatiaia (58242000) até Anta (58630002). Os resultados das correlações são apresentados nas Figuras 7 a 9.

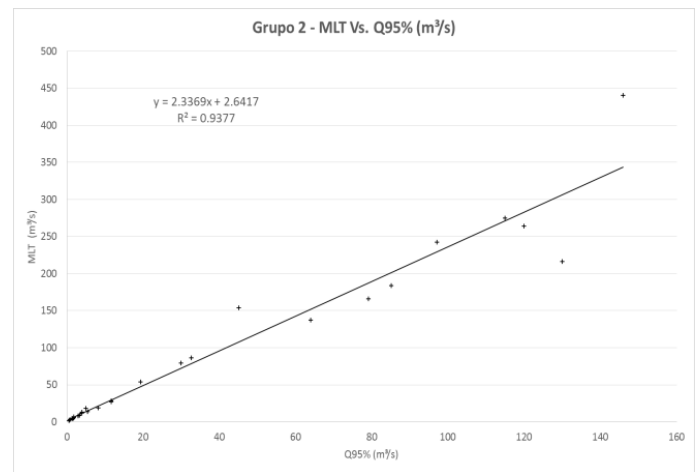

Figura 7. MLT versus Q95\% para o grupo 2 


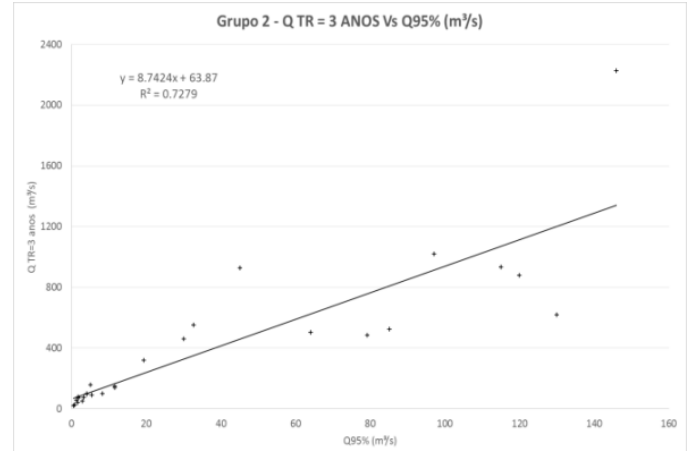

Figura 8. Correlação entre a QTR=3anos e a Q95\% para o grupo 2

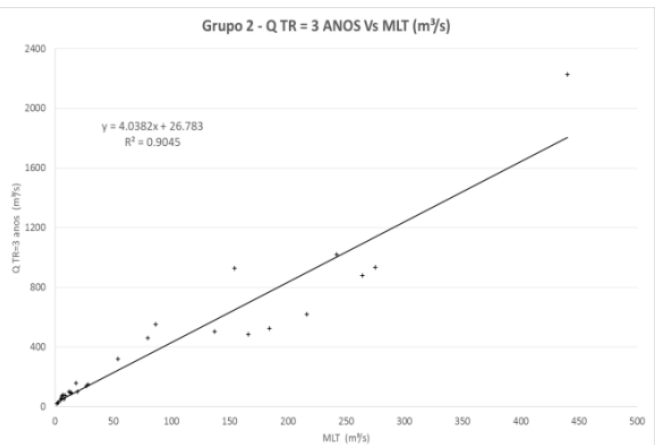

Figura 9. Correlação entre a QTR= 3 anos e a MLT para o grupo 2.

No grupo 3 foram incluídos os postos fluviométricos Cambucas (58837000) até Campos - Ponte Municipal (58974000). Os resultados das correlações são apresentados nas Figuras 10 a 12.

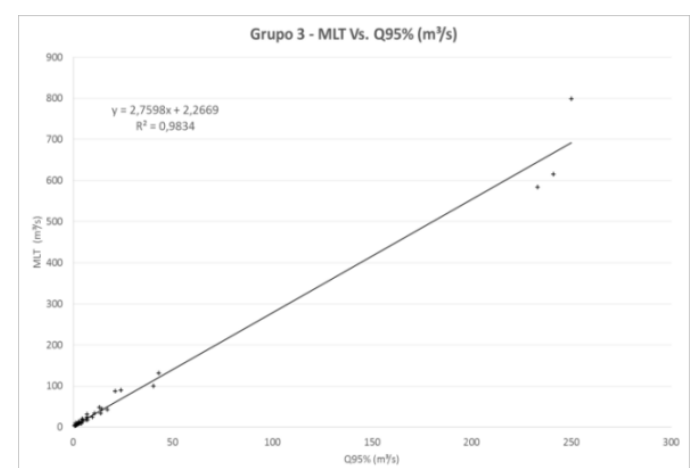

Figura 10. MLT versus Q95\% para o grupo 3

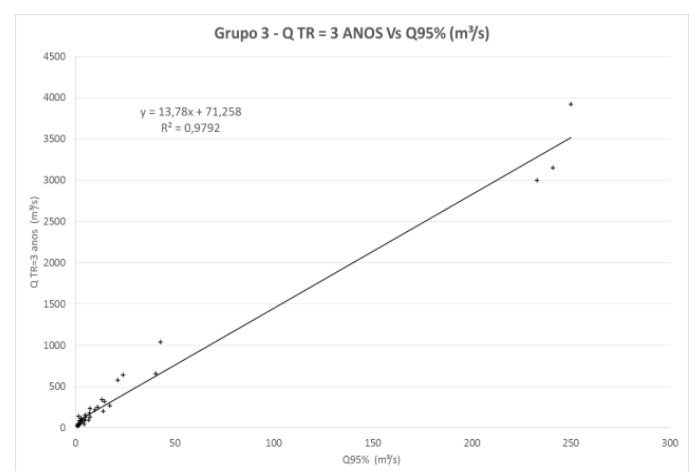

Figura 11. Correlação entre a QTR=3anos e a Q95\% para o grupo 3 


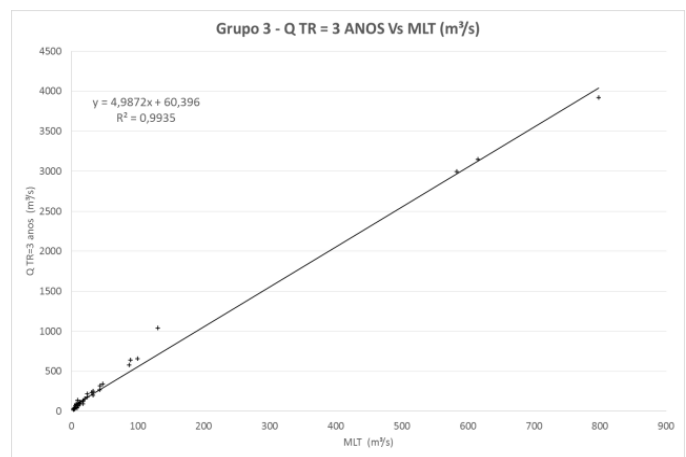

Figura 12. Correlação entre a QTR= 3 anos e a MLT para o grupo 3.

\section{Conclusões e recomendações}

A região da bacia hidrográfica do rio Paraíba do Sul é de extrema importância, pois é o elo entre as maiores regiões metropolitanas do Brasil. Suas águas são um recurso fundamental para diferentes usuários, mas a crescente degradação dos recursos hídricos amplia os conflitos pela água na bacia.

Como estabelecido pela Secretaria do Patrimônio da União, a demarcação das faixas marginais de proteção pode ser feita pela determinação da posição da LMEO, que considera as cotas máximas anuais referentes às enchentes com período de recorrência igual a 3 anos.

No presente estudo, foi possível a obtenção de equações que permitem o cálculo da QTR=3 anos para a bacia do Paraíba do Sul através da sua correlação com as vazões Q95\% e MLT. Da análise dos resultados, podem ser inferidas as seguintes conclusões:

- A vazão Q95\% se relaciona muito bem com a MLT.

- A QTR=3 anos se relaciona melhor com a MLT do que com a Q95\%. Isto já era de se esperar, pois ambas as vazões representam uma condição favorável de vazão, uma a média e a outra a cheia ordinária.

- A divisão do rio Paraíba do Sul em três trechos resulta em melhores ajustes de correlação para o trecho inferior (bacias do rio Pomba, Muriaé e Dois Rios).

- O melhor resultado global foi aquele que leva em consideração todos os postos fluviométricos, sem divisão por trechos.

\section{Referências bibliográficas}

Agência Nacional de Águas (ANA), Bacias do rio Paraíba do Sul. Disponível em: <http://www2.ana.gov.br/Paginas/servicos/outorgaefiscalizacao/RioParaibadoSul.aspx >. Acesso em 11 de junho de 2015.

Eletrobras, Diretrizes para Estudos e Projetos de Pequenas Centrais Hidrelétricas, Capítulo 6: Estudos Básicos, Disponível em: <http://www.eletrobras.gov.br/elb/portal/data/Pages/LUMISF99678B3PTBRIE.htm>. Acessado em fevereiro de 2015. 
MARENGO, José A.; ALVES, Lincoln Muniz, Tendências Hidrológicas da Bacia do rio Paraíba do Sul. Revista Brasileira de Meteorologia. V.20. n.2. 215-226. 2005.

MIRANDA. Anaiza Helena Malhardes. APP em área urbana consolidada. Boletim Eletrônico IRIB. São Paulo. $n^{\circ}$ 3230. 2008. Disponível em: <http://www.irib.org.br/be/BE3230.html>. Acessado em Fevereiro de 2015.

MIRANDA. Ana Carolina Cabral. As legislações federais e estaduais referentes à faixa marginal de proteção: a nova ótica. 2013. 47f. Trabalho de Conclusão de Curso (Graduação em Engenharia Ambiental) - Escola de Engenharia. Universidade Federal Fluminense. Niterói. Rio de Janeiro. 2013.

OLIVEIRA, Ricardo Castro Nunes; MIGUEZ, Marcelo Gomes . O Domínio dos Terrenos Marginais e seu impacto na Requalificação Fluvial. In: XIX Simpósio Brasileiro de Recursos Hídricos. Maceió. Alagoas. 2011.

SERVIÇO GEOLÓGICO DO BRASIL (CPRM). Projeto "Disponibilidade Hídrica do Brasil Estudos de Regionalização nas Bacias Hidrográficas Brasileiras”. Regionalização da Q95\% da Sub-Bacia 58. Relatório. Rio de Janeiro. 96p. 2013.

Secretaria do Patrimônio da União (SPU). Orientação Normativa ON-GEADE-003. Orientação normativa que disciplina a demarcação de terrenos marginais e seus acrescidos. Secretaria do Patrimônio da União. Distrito Federal. Brasília. 2001. Disponível em: <http://patrimoniodetodos.gov.br/pastaarquivo.2009-07-

09.3759851862/ON_GEADE_03_terrenos_marginais-1.pdf >. Acessado em Janeiro de 2013. 
ANEXO

Tabela 1. Resultados das vazões Q95\%, MLT e QTR=3 anos.

\begin{tabular}{|c|c|c|c|c|}
\hline Região & Posto & Q $95 \%\left(\mathrm{~m}^{3} / \mathrm{s}\right)$ & $\operatorname{MLT}\left(\mathrm{m}^{3} / \mathbf{s}\right)$ & $\mathrm{Q}$ TR $=3\left(\mathrm{~m}^{3} / \mathrm{s}\right)$ \\
\hline \multirow[t]{3}{*}{ Região 1} & 58030000 & 5,90 & 12,60 & 51,69 \\
\hline & 58040000 & 13,90 & 30,40 & 147,28 \\
\hline & 58045000 & 15,70 & 35,90 & 176,24 \\
\hline Região 2 & 58183000 & 74,00 & 152,00 & 430,71 \\
\hline \multirow[t]{2}{*}{ Região 3} & 58082000 & 4,16 & 7,93 & 35,85 \\
\hline & 58087000 & 14,40 & 31,20 & 386,54 \\
\hline \multirow[t]{5}{*}{ Região 4} & 58060000 & 3,48 & 8,44 & 144,90 \\
\hline & 58065000 & 0,37 & 1,57 & 40,08 \\
\hline & 58070000 & 8,12 & 21,30 & 460,87 \\
\hline & 58075000 & 0,93 & 2,07 & 17,18 \\
\hline & 58078000 & 10,80 & 25,50 & 321,71 \\
\hline \multirow[t]{6}{*}{ Região 5} & 58099000 & 34,80 & 77,60 & 227,98 \\
\hline & 58107100 & 0,20 & 0,57 & 7,62 \\
\hline & 58110002 & 38,60 & 61,80 & 154,45 \\
\hline & 58170000 & 1,51 & 3,64 & 33,77 \\
\hline & 58207000 & 1,04 & 3,34 & 24,68 \\
\hline & 58220000 & 1,36 & 3,57 & 38,94 \\
\hline \multirow[t]{4}{*}{ Região 6} & 58142200 & 4,04 & 9,47 & 50,96 \\
\hline & 58235100 & 93,10 & 214,00 & 723,29 \\
\hline & 58242000 & 130,00 & 216,00 & 619,51 \\
\hline & 58250000 & 97,10 & 242,00 & 1020,82 \\
\hline \multirow[t]{5}{*}{ Região 7} & 58118000 & 2,91 & 7,77 & 50,69 \\
\hline & 58122000 & 5,40 & 13,80 & 88,89 \\
\hline & 58126000 & 8,25 & 19,00 & 98,58 \\
\hline & 58128000 & 11,60 & 28,20 & 147,18 \\
\hline & 58129000 & 11,60 & 27,00 & 137,87 \\
\hline \multirow[t]{2}{*}{ Região 8} & 58204000 & 79,20 & 166,00 & 485,78 \\
\hline & 58218000 & 85,10 & 184,00 & 525,34 \\
\hline \multirow[t]{4}{*}{ Região 9} & 58258000 & 1,78 & 6,81 & 78,84 \\
\hline & 58287000 & 1,82 & 5,72 & 72,27 \\
\hline & 58300000 & 120,00 & 264,00 & 880,23 \\
\hline & 58305001 & 115,00 & 275,00 & 933,22 \\
\hline \multirow[t]{5}{*}{ Região 10} & 58256000 & 0,52 & 1,60 & 21,64 \\
\hline & 58525000 & 1,34 & 5,09 & 57,72 \\
\hline & 58530000 & 4,00 & 13,30 & 95,98 \\
\hline & 58535000 & 5,03 & 18,02 & 158,54 \\
\hline & 58550001 & 19,30 & 54,03 & 319,98 \\
\hline \multirow[t]{4}{*}{ Região 11} & 58270000 & 3,23 & 8,80 & 76,00 \\
\hline & 58542000 & 3,98 & 11,90 & 100,95 \\
\hline & 58560000 & 0,84 & 2,77 & 25,70 \\
\hline & 58573000 & 1,58 & 4,39 & 41,64 \\
\hline
\end{tabular}


ISSN: $1415-7314$

ISSN online: $2317-6717$

\begin{tabular}{|c|c|c|c|c|}
\hline & 58585000 & 30,00 & 79,60 & 460,60 \\
\hline & 58590000 & 32,70 & 86,40 & 551,33 \\
\hline \multirow[t]{4}{*}{ Região 12} & 58380001 & 45,10 & 154,00 & 928,81 \\
\hline & 58385000 & 64,00 & 137,00 & 502,37 \\
\hline & 58630002 & 146,00 & 440,00 & 2225,82 \\
\hline & 58837000 & 0,98 & 4,06 & 44,65 \\
\hline \multirow[t]{10}{*}{ Região 13} & 58470000 & 3,48 & 7,92 & 40,53 \\
\hline & 58480500 & 9,68 & 21,50 & 156,37 \\
\hline & 58500000 & 1,36 & 3,05 & 29,83 \\
\hline & 58512000 & 16,20 & 36,70 & 183,76 \\
\hline & 58516000 & 20,40 & 53,10 & 242,89 \\
\hline & 58516500 & 19,60 & 48,00 & 255,54 \\
\hline & 58519000 & 34,60 & 80,30 & 387,32 \\
\hline & 58520000 & 30,80 & 75,70 & 349,80 \\
\hline & 58610000 & 5,30 & 14,50 & 89,58 \\
\hline & 58620000 & 71,00 & 184,00 & 870,70 \\
\hline \multirow[t]{4}{*}{ Região 14} & 58400000 & 0,77 & 2,49 & 60,64 \\
\hline & 58405000 & 3,17 & 11,10 & 134,70 \\
\hline & 58409000 & 3,69 & 12,50 & 107,51 \\
\hline & 58440000 & 8,16 & 37,70 & 439,43 \\
\hline \multirow[t]{9}{*}{ Região 15} & 58420000 & 5,63 & 16,90 & 160,31 \\
\hline & 58427000 & 8,66 & 22,40 & 137,31 \\
\hline & 58820000 & 1,42 & 4,62 & 26,71 \\
\hline & 58825000 & 2,38 & 7,50 & 84,73 \\
\hline & 58830000 & 1,24 & 4,18 & 34,33 \\
\hline & 58832000 & 1,62 & 5,41 & 48,87 \\
\hline & 58846000 & 9,77 & 23,90 & 216,52 \\
\hline & 58850000 & 11,00 & 32,60 & 247,78 \\
\hline & 58861000 & 1,04 & 2,74 & 25,96 \\
\hline \multirow[t]{5}{*}{ Região 16} & 58645000 & 2,04 & 5,68 & 49,35 \\
\hline & 58648001 & 4,18 & 11,70 & 98,89 \\
\hline & 58857000 & 2,04 & 5,54 & 42,09 \\
\hline & 58870000 & 3,14 & 10,50 & 93,46 \\
\hline & 58874000 & 14,70 & 43,00 & 314,62 \\
\hline \multirow[t]{8}{*}{ Região 17} & 58710000 & 7,37 & 17,60 & 132,65 \\
\hline & 58720000 & 3,47 & 8,27 & 62,11 \\
\hline & 58725000 & 4,37 & 8,51 & 43,38 \\
\hline & 58730001 & 14,00 & 32,70 & 198,26 \\
\hline & 58735000 & 17,40 & 42,40 & 265,22 \\
\hline & 58736000 & 1,45 & 9,37 & 139,10 \\
\hline & 58750000 & 4,92 & 13,10 & 92,56 \\
\hline & 58755000 & 6,62 & 17,40 & 93,03 \\
\hline \multirow[t]{3}{*}{ Região 18} & 58658000 & 1,66 & 4,25 & 34,44 \\
\hline & 58670002 & 2,72 & 7,02 & 89,13 \\
\hline & 58765001 & 7,24 & 30,50 & 232,89 \\
\hline
\end{tabular}

ENGEVISTA, V. 19, n.1 , p. 55-67, Janeiro 2017. 


\begin{tabular}{ccccc} 
& 58770000 & 40,50 & 100,00 & 655,43 \\
\hline Região 19 & 58790000 & 43,00 & 131,00 & 1039,97 \\
\hline & 58910000 & 0,94 & 3,30 & 19,39 \\
\hline & 58915000 & 7,09 & 23,70 & 178,89 \\
\hline Região 20 & 58916000 & 2,90 & 9,99 & 64,56 \\
\hline & 58917000 & 4,85 & 17,20 & 127,73 \\
\hline & 58795000 & 233,00 & 583,00 & 2994,75 \\
\hline & 58880001 & 241,00 & 615,00 & 3147,55 \\
\hline & 58920000 & 13,40 & 47,50 & 341,65 \\
\hline & 58930000 & 2,95 & 12,90 & 113,61 \\
\hline & 58934000 & 4,80 & 19,70 & 153,81 \\
\hline & 58940000 & 24,10 & 89,40 & 637,04 \\
\hline & 58960000 & 21,30 & 87,60 & 574,11 \\
\hline & 58974000 & 250,00 & 798,00 & 3916,89 \\
\hline & 58335000 & 1,45 & 4,53 & 67,15 \\
\hline & 58338000 & 2,10 & 8,19 & 118,03 \\
\hline & 58350001 & 0,71 & 5,89 & 92,92 \\
\hline & 58359000 & 1,00 & 4,58 & 69,35 \\
\hline
\end{tabular}

